\title{
MULTIPLICITIES OF ZEROES OF POLYNOMIALS ON TRAJECTORIES OF POLYNOMIAL VECTOR FIELDS AND BOUNDS ON DEGREE OF NONHOLONOMY
}

\author{
Andrei Gabrielov
}

\begin{abstract}
A вsтRAст. The multiplicity of a zero of a restriction of a polynomial of degree $q$ in $\mathbb{C}^{n}, n \geq 2$, to a non-singular trajectory of a polynomial vector field $\xi$ with coefficients of degree $p$ does not exceed $[p q(p+q)]^{2^{n-2}}$ if the polynomial does not vanish identically on this trajectory. For a system of polynomial vector fields, this implies an effective estimate on degree of nonholonomy, i.e., the minimal order of brackets necessary to generate a subspace of maximal possible dimension at each point. In particular, this allows one to check effectively whether a given system of polynomial vector fields is totally nonholonomic (controllable) at each point. Similar estimates are found for systems of vector fields with analytic coefficients satisfying polynomial Pfaffian equations. This allows one to check effectively whether such a system is totally nonholonomic (controllable) at a given point.
\end{abstract}

\section{Introduction}

The problem of an effective bound on the multiplicity of a zero of a polynomial $Q$ in $n$ variables restricted to a trajectory of a polynomial vector field $\xi$ was suggested by J. J. Risler [18] in connection with the problem of controllability of polynomial control systems in nonholonomic control theory [4, 21]. For $n=2$, the solution is given in [6]. In this case, the problem is a special case of the estimates for the Pfaffian multiplicities [7]. However, for $n \geq 3$, the problem cannot be reduced to the Pfaffian case. J. J. Risler [18] found that the order at $z$ of the restriction of $Q$ to a trajectory of $\xi$ through $z$ does not exceed $\mu+n-1$ where $\mu$ is the multiplicity at $z$ of the ideal generated by the polynomial $Q$ and its $n-1$

Received April 19, 1995.

Supported in part by the U.S. Army Research Office through the Army Center of Excellence for Symbolic Methods in Algorithmic Mathematics (ACSyAM), Mathematical Sciences Institute of Cornell University, Contract DAAL03-91-C-0027. 
consecutive derivatives along $\xi$ at $z$. If $\mu$ is finite, it can be easily estimated through Bezout's theorem. The estimate is single exponential in $n$.

In this paper, we give an effective estimate of the multiplicity $\kappa$ of a zero of an arbitrary polynomial of degree $q$ on a trajectory of a polynomial vector field with coefficients of degree $p$ in $\mathbb{C}^{n}$ (Theorem 1 ):

$$
\kappa \leq p^{2^{n-2}-1} q^{2^{n-2}}(p+q)^{2^{n-2}} .
$$

This estimate is doubly exponential in $n$.

For a system of polynomial vector fields of degree $p$ in $\mathbb{C}^{n}$, the estimate (1) allows to define an effective upper bound on the degree of nonholonomy, i.e., the minimal order $N$ of brackets necessary to generate a subspace of maximal possible dimension $d$ at each point (Theorem 3 ):

$$
N \leq \begin{cases}(d-2)\left(2^{3 d-7} 5 p^{3}\right)^{2^{n-2}}+d-1, & \text { for } d \geq 3 \\ \left(6 p^{3}\right)^{2^{n-2}}+1, & \text { for } d=2\end{cases}
$$

Obviously, $N=1$ for $d=1$.

In particular, a system of polynomial vector fields is totally nonholonomic, i.e., $d=n$ at each point, when a finite system of polynomial equations defined by coefficients of all brackets of order not exceeding $N$ is incompatible. The latter property can be checked (at least for polynomials with rational coefficients) with any standard algorithm for deciding consistency of a system of algebraic equations. Thus, the problem whether a system of polynomial vector fields is totally nonholonomic is algorithmically decidable (Corollary from Theorem 3).

The estimates for vector fields with "Noetherian" coefficients, i.e., analytic functions satisfying polynomial Pfaffian equations, can be easily deduced from the estimates (1) and (2) for polynomial vector fields (Theorems 2 and 4). In order to check whether a given system of vector fields with Noetherian coefficients is totally nonholonomic, we have to decide whether a system of transcendental equations with Noetherian functions is compatible. Usually this cannot be done algorithmically. However, if we want to decide whether our system is controllable at a given point where its coefficients are computable, the problem is again reduced to compatibility of a system of algebraic equations, which is algorithmically decidable (Corollary from Theorem 4).

Totally nonholonomic systems play important role in control theory, where they are called controllable $[4,21]$. The estimate (2) allows one to 
check effectively controllability of a given polynomial control system (see [18] for a discussion).

Systems of this kind appear also in the theory of hypoelliptic partial differential equations where the total nonholonomy is known as the "Hörmander's condition," and the degree of nonholonomy is involved in the a priori subelliptic estimates. This connection was first established by Hörmander [12] (see [5, 11, 16, 19, and 13, Ch. 22] for further developments).

In the theory of probabilities, Hörmander's condition appears in connection with the diffusion processes along trajectories of vector fields (Stratonovich diffusion). See [15] for discussion and further references.

In differential geometry, totally nonholonomic systems appear as anisotropic Carnot-Carathéodory [9, 10] or sub-Riemannian [20] metrics. The degree of nonholonomy determines how far the metric is from a Riemannian metric; for a system with degree of nonholonomy $r$, in the worst direction, the Carnot-Carathéodory distance is proportional to $1 / r$-th power of the Riemannian distance.

In the theory of several complex variables, the degree of nonholonomy is closely connected to the 'type' condition for boundaries of non-strictly pseudoconvex domains introduced by Kohn [14], and studied in [2, 3]. See [1] for the latest development in this area.

\section{Polynomials on trajectories of polynomial vector fields}

Lemma 1. Let $Q(x)$ be a germ of an analytic function at $\mathbf{0} \in \mathbb{C}^{n}$, such that

$$
\left.Q\right|_{\left\{x_{1}=\ldots=x_{n-1}=0\right\}} \not \equiv 0 .
$$

Let $Q^{(i)}=\partial^{i} Q / \partial x_{n}^{i}$, and let $I_{i}$ be the ideal generated by $Q^{(0)}, \ldots, Q^{(i)}$ in $\mathbb{C}\{x\}$. Suppose that $Z_{i}=V\left(I_{i}\right)$ has dimension $d$, and let $\mu_{i}$ be the multiplicity of $I_{i}$ at a generic point $z$ of any d-dimensional irreducible component of $Z_{i}$, i.e., the rank at $z$ of $\mathcal{O}_{z}\left(\mathbb{C}^{n}\right) / I_{i}$ over $\mathcal{O}_{z}\left(Z_{i}\right)$. Then $\operatorname{dim} Z_{i+\mu_{i}}<d$.

Proof. Let $z$ be a generic point of a $d$-dimensional component of $Z_{i}$. Due to (3) the direction of the $x_{n}$ axis is not tangent to $Z_{i}$ at $z$. Consider a surface $S$ of codimension $d$, transversal to $Z_{i}$ at $z$, containing the line $L$ parallel to the $x_{n}$ axis through $z$. Let $\nu$ be the order of $\left.Q\right|_{L}$ at $z$. We have $\mu_{i}=\operatorname{dim}_{\mathbb{C}} \mathcal{O}_{z}(S) /\left(I_{i} \otimes \mathcal{O}_{z}(S)\right)$. At the same time, all the generators of $I_{i}$, when restricted to $L$, belong to $\left(t^{\nu-i}\right)$ where $t$ is a local parameter on $L$ at $z$. Hence the epimorphism of rings $\mathcal{O}_{z}(S) \rightarrow \mathbb{C}\{t\}$ (the restriction from $S$ to $L)$ induces an epimorphism of rings $\mathcal{O}_{z}(S) /\left(I_{i} \otimes \mathcal{O}_{z}(S)\right) \rightarrow \mathbb{C}\{t\} /\left(t^{\nu-i}\right)$. 
As the dimension over $\mathbb{C}$ of the second ring is equal to $\nu-i$, we have $\nu-i \leq \mu_{i}$. This means that the point $z$ cannot belong to $Z_{i+\mu_{i}}$.

Theorem 1. Let $\xi(y)=\sum_{j} P_{j}(y) \partial / \partial y_{j}$ be a vector field in $\mathbb{C}^{n}, n \geq 2$, with polynomial in $y=\left(y_{1}, \ldots, y_{n}\right)$ coefficients $P_{j}$ of degree not exceeding $p \geq 1$. Let $\gamma$ be a non-singular trajectory of $\xi$, i.e., $\xi \neq 0$ at each point of $\gamma$. For a polynomial $Q(y)$ of degree $q$, either $\left.Q\right|_{\gamma} \equiv 0$ or the order of a zero of $\left.Q\right|_{\gamma}$ at each point of $\gamma$ does not exceed

$$
p^{2^{n-2}-1} q^{2^{n-2}}(p+q)^{2^{n-2}} .
$$

Proof. Let $y_{0}$ be a point of $\gamma$ where (4) is violated. Let us choose the coordinates $x$ in $\mathbb{C}^{n}$ near $y_{0}$ so that $\xi=\partial / \partial x_{n}$, and use the notations of Lemma 1 in these coordinates. Then $Q^{(i)}$ is the $i$-th derivative of $Q$ along $\xi$. Suppose that $\left.Q\right|_{\gamma} \not \equiv 0$. Then $Q \not \equiv 0$, hence $\operatorname{dim} Z_{0}=n-1$. We can suppose also that $Q$ is irreducible, because the estimate for $Q_{1} Q_{2}$ follows from the estimates for $Q_{1}$ and $Q_{2}$. In this case, the multiplicity of $Q$ at a generic point of $Z_{0}$ is equal to 1 . From Lemma 1 we have $\operatorname{dim} Z_{1}=$ $n-2$. To estimate the multiplicity $\mu_{1}$, we note that $Q^{(1)}=\sum_{j} P_{j} \partial Q / \partial y_{j}$ is a polynomial in $y$ of degree not exceeding $q+p-1$. From Bezout's theorem applied to $Q$ and $Q^{(1)}$ restricted to a 2-dimensional linear (in the coordinates $y$ ) subspace transversal to $Z_{1}$ in its generic point, $\mu_{1} \leq$ $q(q+p-1) \leq q(p+q)-1$. From Lemma 1 we have $\operatorname{dim} Z_{q(p+q)}<n-2$. Let us choose $\nu \leq q(p+q)$ so that $\operatorname{dim} Z_{\nu}=n-3$. For a generic linear combination $G$ of $Q^{(2)}, \ldots, Q^{(\nu)}$, the functions $Q, Q^{(1)}$, and $G$ define a complete intersection $Y$ of dimension $n-3$. The function $G$ is a polynomial in $y$ of degree not exceeding $q+(p-1) q(p+q)$. Let $L$ be a linear (in the coordinates $y$ ) 3 -dimensional subspace transversal to $Y$ in its generic point $z$, and let $I$ be the ideal generated in $\mathcal{O}_{z}(L)$ by the restrictions of $Q, Q^{(1)}$, and $G$ to $L$. From Bezout's theorem $\operatorname{dim}_{\mathbb{C}} \mathcal{O}_{z}(L) / I$ does not exceed $q(p+q-1)[q+(p-1) q(p+q)] \leq p q^{2}(p+q)^{2}-q(p+q)$. Hence $\mu_{\nu}$ does not exceed $p q^{2}(p+q)^{2}-q(p+q)$. From Lemma 1 we have $\operatorname{dim} Z_{p q^{2}(p+q)^{2}}<n-3$.

The same argument inductively shows that

$$
\operatorname{dim} Z_{\nu}<n-k, \text { for } 2 \leq k \leq n \text { and } \nu \geq p^{2^{k-2}-1} q^{2^{k-2}}(p+q)^{2^{k-2}} .
$$

Specifically, for $\nu \geq p^{2^{n-2}-1} q^{2^{n-2}}(p+q)^{2^{n-2}}, \operatorname{dim} Z_{\nu}<0\left(Z_{\nu}\right.$ is empty). 
Definition 1. (Khovanskii, unpublished; see Tougeron [22].) A Noetherian chain of order $m$ and degree $\alpha$ is a system $\mathbf{f}=\left(f_{1}(y), \ldots, f_{m}(y)\right)$ of germs of analytic functions at the origin $\mathbf{0}$ of a complex or real $n$ dimensional space, satisfying Pfaffian equations

$$
\frac{\partial f_{i}}{\partial y_{j}}=g_{i j}\left(y, f_{1}(y), \ldots, f_{m}(y)\right), \text { for } i=1, \ldots, m \text { and } j=1, \ldots, n,
$$

where $g_{i j}$ are polynomials in $y$ and $f$ of degree not exceeding $\alpha \geq 1$. A function $\phi(y)=P\left(y, f_{1}(y), \ldots, f_{m}(y)\right)$, where $P$ is a polynomial in $y$ and $f$ of degree not exceeding $p$, is called a Noetherian function of degree $p$, with the Noetherian chain $f_{1}, \ldots, f_{m}$. For the real case, all the functions and polynomials in this definition are supposed to be real.

Theorem 1 can be extended from polynomial to Noetherian functions as follows:

Theorem 2. Let $\mathbf{f}=\left(f_{1}, \ldots, f_{m}\right)$ be a Noetherian chain of order $m$ and degree $\alpha$, and let $\xi=\sum_{j} \phi_{j}(y) \partial / \partial y_{j}$ be a vector field with the coefficients $\phi_{j}$ Noetherian of degree $p$, with the Noetherian chain $\mathbf{f}$. Let $\psi$ be a Noetherian function of degree $q$, with the Noetherian chain $\mathbf{f}$. Suppose that $\xi(\mathbf{0}) \neq 0$ and that $\psi$ does not vanish identically on the trajectory $\gamma$ of $\xi$ through $\mathbf{0 .}$ Then the multiplicity of the zero of $\left.\psi\right|_{\gamma}$ at $\mathbf{0}$ does not exceed

$$
(p+\alpha)^{2^{m+n-2}-1} q^{2^{m+n-2}}(p+\alpha+q)^{2^{m+n-2}} .
$$

Proof. The statement follows from Theorem 1 after we add $f_{1}, \ldots, f_{m}$ to the list of variables and note that $\left.\psi\right|_{\gamma}$ coincides with the restriction of a polynomial $Q(y, f)$ to the trajectory through $\mathbf{0}$ of the polynomial vector field

$$
\sum_{j=1}^{n} P_{j}(y, f) \frac{\partial}{\partial y_{j}}+\sum_{i=1}^{m}\left[\sum_{j=1}^{n} P_{j}(y, f) g_{i j}(y, f)\right] \frac{\partial}{\partial f_{i}}
$$

Remark. Simple arguments (see [22]) show that, for any given integers $n, m, \alpha$, and $q$, there exists an integer $M(n, m, \alpha, q)$ such that the multiplicity of any isolated intersection $\psi_{1}(y)=\ldots=\psi_{n}(y)=0$, where $\psi_{j}$ are Noetherian functions in $\mathbb{C}^{n}$ of degree $q$, with a Noetherian chain of order $m$ and degree $\alpha$, does not exceed $M(n, m, \alpha, q)$.

To show this, we first recall that the condition $\mu \geq M$, for the multiplicity $\mu$ of an intersection defined by analytic functions $\psi_{i}$ in $\mathbb{C}^{n}$, can 
be formulated as a system of polynomial equations on the values of the functions $\psi_{i}$ and their partial derivatives of order not exceeding $\mu_{0}$. For Noetherian functions $\psi_{j}$, the values of their partial derivatives can be expressed as polynomials of the variables $y_{i}$, values of $\psi_{j}$, and the coefficients of the polynomials involved in the definition of the Noetherian chain and function. Consider now the ring $R$ of polynomials in all these variables. Condition $\mu \geq M$ is represented now by an ideal $I_{M} \subset R$. As $I_{M} \subseteq I_{M+1}$, for each $M$, and $R$ is a Noetherian ring, the sequence $I_{M}$ stabilizes at some $M=M(n, m, \alpha, q)$. This means that any intersection with the multiplicity $\mu=M(m, n, \alpha, q)$ or greater has infinite multiplicity.

Theorem 1 can be interpreted now as an explicit estimate of the number $M(1, n, p, q)$ for Noetherian functions in one variable. The corresponding effective estimate in $n>1$ variables remains an open problem.

\section{Degree of nonholonomy}

Definition 2. For a set $\Xi$ of analytic vector fields $\xi_{i}$ in $\mathbb{C}^{n}$, let $\mathcal{L}_{1}(\Xi)$ denote the space of all linear combinations of $\xi_{i}$ with complex coefficients. For $r \geq 2$, we define $\mathcal{L}_{r}(\Xi)=\mathcal{L}_{r-1}(\Xi)+\left[\mathcal{L}_{1}(\Xi), \mathcal{L}_{r-1}(\Xi)\right]$. Finally, $\mathcal{L}(\Xi)=$ $\cup_{r} \mathcal{L}_{r}(\Xi)$ is the Lie algebra generated by the vector fields $\xi_{i}$. For $z \in \mathbb{C}^{n}$, let $d_{r}(z)$ be dimension of the subspace generated by the values at $z$ of the vector fields from $\mathcal{L}_{r}(\Xi)$, and let $d(\Xi, z)=\max _{r} d_{r}(z)$ be dimension of the subspace generated by the values at $z$ of the vector fields from $\mathcal{L}(\Xi)$. The minimal $r$ such that $d_{r}(z)=d(\Xi, z)$ is called degree of nonholonomy of $\Xi$ at $z$. In particular, $d(\Xi, z)=n$ means that $\Xi$ is totally nonholonomic at $z$ (see $[8,23])$. It is easy to check that the values $d_{r}$ do not change if we allow linear combinations of vector fields with analytic (instead of constant) coefficients. The same is true for degree of nonholonomy.

Definition 3. For a germ at $\mathbf{0}$ of an analytic vector field $\xi$ in $\mathbb{C}^{n}$ such that $\xi(\mathbf{0}) \neq 0$, let $\gamma(\xi)$ denote a germ at $\mathbf{0}$ of the trajectory of $\xi$ through $\mathbf{0}$. For a finite set $\left\{\xi_{1}, \ldots, \xi_{k}\right\}$ of germs at $\mathbf{0}$ of analytic vector fields in $\mathbb{C}^{n}$, let $\epsilon=\left(\epsilon_{1}, \ldots, \epsilon_{k}\right) \in \mathbb{C}^{k}$ and $\xi_{\epsilon}=\xi+\epsilon_{1} \xi_{1}+\ldots+\epsilon_{k} \xi_{k}$. Let $\Xi(x, \epsilon)=\left(\xi_{\epsilon}(x), 0\right)$ be a germ at $\left(\mathbf{0}, \mathbf{0}_{\epsilon}\right)$ of a vector field in $\mathbb{C}^{n} \times \mathbb{C}^{k}$. The flow of $\Xi$ through $\mathbf{0} \times \mathbb{C}^{k}$ generates a germ at $\left(\mathbf{0}, \mathbf{0}_{\epsilon}\right)$ of a $(k+1)$-dimensional analytic manifold $B=B\left(\xi, \xi_{1}, \ldots, \xi_{k}\right)$. This manifold can be considered as a union of germs $\left(\gamma\left(\xi_{\epsilon}\right), \epsilon\right)$ over small values of $\epsilon \in \mathbb{C}^{k}$.

Let $\pi: \mathbb{C}^{n} \times \mathbb{C}^{k} \rightarrow \mathbb{C}^{n}$. A value of $\epsilon$ is called regular if the rank of $\pi: B \rightarrow \mathbb{C}^{n}$ is equal $k+1$ at small nonzero points of $\left(\gamma\left(\xi_{\epsilon}\right), \epsilon\right)$. The system 
of vector fields $\xi, \xi_{1}, \ldots, \xi_{k}$ is called non-degenerate if the set of regular values of $\epsilon$ is non-empty.

Note that $B$ remains essentially unchanged when we replace $\xi$ by $\xi_{\epsilon}$, for a small $\epsilon \in \mathbb{C}^{k}$. More precisely, if we choose a representative $\tilde{B}$ of the germ $B$ in $\mathbb{C}^{n} \times \mathbb{C}^{k}$ and choose $\epsilon$ so that $(\mathbf{0}, \epsilon) \in \tilde{B}$, then the germ $B\left(\xi_{\epsilon}, \xi_{1}, \ldots, \xi_{k}\right)$ at the origin of $\mathbb{C}^{n} \times \mathbb{C}^{k}$ coincides with the germ of $\tilde{B}$ at $(\mathbf{0}, \epsilon)$.

Lemma 2. For a small $\epsilon \in \mathbb{C}^{k}$ and a small nonzero point $z \in \gamma\left(\xi_{\epsilon}\right)$, the tangent space $T_{z, \epsilon} B$ to $B=B\left(\xi, \xi_{1}, \ldots, \xi_{k}\right)$ at $(z, \epsilon)$ is generated by the vectors $\left(\zeta_{i}(z), \mathbf{1}_{i}\right)$, for $i=1, \ldots, k$, and $\left(\xi_{\epsilon}(z), \mathbf{0}_{\epsilon}\right)$. Here $\mathbf{1}_{i}=\partial / \partial \epsilon_{i}$ is the $i$-th basis vector in $\mathbb{C}^{k}$, and the vector fields $\zeta_{i}$ satisfy the following conditions: $\zeta_{i}(\mathbf{0})=0$ and $\left[\xi_{\epsilon}, \zeta_{i}\right]=\xi_{i}$.

Proof. In a coordinate system where $\xi_{\epsilon}=\partial / \partial x_{1} \equiv(1,0, \ldots, 0)$ we have $\left[\xi_{\epsilon}, \zeta_{i}\right]=\partial \zeta_{i} / \partial x_{1}$ and $\gamma\left(\xi_{\epsilon}\right)$ coincides with the $x_{1}$-axis. Let $z=(t, 0, \ldots, 0)$ be the point of $\gamma\left(\xi_{\epsilon}\right)$ at a time moment $t$. Let $\mathbf{g}_{i}\left(t, \epsilon_{i}\right)$ be the point of $\gamma\left(\xi_{\epsilon}+\epsilon_{i} \xi_{i}\right)$ at a time moment $t$. We have $\mathbf{g}_{i}\left(0, \epsilon_{i}\right) \equiv 0$ and $\mathbf{g}_{i}(t, 0)=z$. As the trajectories $\gamma\left(\xi_{\epsilon}+\epsilon_{i} \xi_{i}\right)$ belong to $B$, its tangent space $T_{z, \epsilon} B$ at a point $(z, \epsilon)$ contains vectors $\left(\partial \mathbf{g}_{i}\left(t, \epsilon_{i}\right) / \partial \epsilon_{i}, \mathbf{1}_{i}\right)$, for $i=1, \ldots, k$, and $\left((1,0, \ldots, 0), \mathbf{0}_{\epsilon}\right)$. As these $k+1$ vectors are independent, they generate $T_{z, \epsilon} B$.

To prove Lemma 2, we want to show that

$$
\zeta_{i}(t, 0, \ldots, 0)=\frac{\partial \mathbf{g}_{i}}{\partial \epsilon_{i}}(t, 0)
$$

By definition, $\partial \mathbf{g}_{i}\left(t, \epsilon_{i}\right) / \partial t=\xi_{\epsilon}\left(g_{i}\left(t, \epsilon_{i}\right)\right)+\epsilon_{i} \xi_{i}\left(g_{i}\left(t, \epsilon_{i}\right)\right)$. As $\xi_{\epsilon}$ is a constant vector field in our coordinate system,

$$
\frac{\partial^{2} \mathbf{g}_{i}}{\partial t \partial \epsilon_{i}}(t, 0)=\xi_{i}(t, 0, \ldots, 0)=\frac{\partial \zeta_{i}}{\partial t}(t, 0, \ldots, 0)
$$

This implies $\zeta_{i}(t, 0, \ldots, 0)=\partial \mathbf{g}_{i} / \partial \epsilon_{i}(t, 0)$, as both sides of this equation vanish at $t=0$.

Lemma 3. For a non-degenerate system $\xi, \xi_{1}, \ldots, \xi_{k}$, the set of regular values of $\epsilon$ is an open semi-algebraic set in $\mathbb{C}^{k}$.

Proof. According to Lemma $2, \epsilon$ is a regular value if and only if $\Omega=$ $\xi_{\epsilon} \wedge \zeta_{1} \wedge \ldots \wedge \zeta_{k}$, does not vanish identically on $\gamma\left(\xi_{\epsilon}\right)$. Let $\epsilon$ be a regular value. As $\Omega$ is analytic on $\gamma_{\epsilon}$, it has a finite multiplicity $\nu$ at the origin. 
This implies that $L_{\xi_{\epsilon}}^{\nu} \Omega$, the $\nu$-th derivative of $\Omega$ along $\xi_{\epsilon}$ does not vanish at the origin. For a non-regular value of $\epsilon$, we have

$$
L_{\xi_{\epsilon}}^{\nu} \Omega(\mathbf{0})=0
$$

for all $\nu \geq 0$. As $\zeta_{i}(\mathbf{0})=0$ and $L_{\xi_{\epsilon}} \zeta_{i}=\xi_{i}$, (7) is valid for all $\epsilon$ when $\nu<k$, and $L_{\xi_{\epsilon}}^{\nu} \Omega(\mathbf{0})$ is polynomial in $\epsilon$ of degree $\nu-k+1$, for $\nu \geq k$. Hence the set of non-regular values of $\epsilon$ is defined by a system of algebraic equations (7) in $\mathbb{C}^{k}$.

Lemma 4. Let $\xi, \xi_{1}, \ldots, \xi_{k}$ be a non-degenerate system of vector fields, $\xi(\mathbf{0}) \neq 0$, and let $\epsilon=\left(\epsilon_{1}, \ldots, \epsilon_{k}\right)$ be a regular value. If $\left[\xi_{\epsilon}, \xi_{i}\right](z)$ belong to the subspace generated by $\xi_{\epsilon}(z), \xi_{1}(z), \ldots, \xi_{k}(z)$, for small nonzero $z \in \gamma\left(\xi_{\epsilon}\right)$ and $i=1, \ldots, k$, then $\xi_{\epsilon}(z), \xi_{1}(z), \ldots, \xi_{k}(z)$ generate $L_{z}=$ $\pi T_{z, \epsilon} B\left(\xi, \xi_{1}, \ldots, \xi_{k}\right)$, for small nonzero $z \in \gamma\left(\xi_{\epsilon}\right)$.

Proof. In a coordinate system where $\xi_{\epsilon}=\partial / \partial x_{1}$, we have $\left[\xi_{\epsilon}, \xi_{i}\right]=\partial \xi_{i} / \partial x_{1}$ and $\gamma\left(\xi_{\epsilon}\right)$ is the $x_{1}$-axis. Let $V(t)$ be the subspace generated by

$$
\xi_{\epsilon}(z), \xi_{1}(z), \ldots, \xi_{k}(z)
$$

at $z=(t, 0, \ldots, 0) \in \gamma\left(\xi_{\epsilon}\right)$. If $\left[\xi_{\epsilon}, \xi_{i}\right](t, 0, \ldots, 0)=d / d t\left(\xi_{i}(t, 0, \ldots, 0)\right)$ belong to $V(t)$, for $i=1, \ldots, k$ and small $t \neq 0$, then $V(t) \equiv V$ does not depend on $t$. Due to Lemma 2, the space $L_{z}$, for $z=(t, 0, \ldots, 0)$, is generated by $(1,0, \ldots, 0)$ and $k$ vectors $\zeta_{i}(t)$ satisfying $\zeta_{i}(0)=0$ and $d / d t\left(\zeta_{i}(t)\right)=\xi_{i}(t, 0, \ldots, 0)$. This implies that $\zeta_{i}(t) \in V$, for small $t$. As $\epsilon$ is a regular value, the $k+1$ vectors $(1,0, \ldots, 0), \zeta_{1}(t), \ldots, \zeta_{k}(t)$ are linearly independent, for small nonzero $t$. As all these vectors belong to $V$, and dimension of $V$ does not exceed $k+1$, the space $V$ coincides with $L_{z}$, for each small nonzero $z=(t, 0, \ldots, 0) \in \gamma\left(\xi_{\epsilon}\right)$. Hence this space is generated by $\xi_{\epsilon}, \xi_{1}, \ldots, \xi_{k}$.

Lemma 5. If the conditions of Lemma 4 are valid for all values of $\epsilon$ in an open set in $\mathbb{C}^{k}$ then $d\left(\xi, \xi_{1}, \ldots, \xi_{k}, \mathbf{0}\right)=k+1$, i.e., there exists a $(k+1)$-dimensional integral submanifold for $\xi, \xi_{1}, \ldots, \xi_{k}$ through $\mathbf{0}$.

Proof. The value of $d=d\left(\xi, \xi_{1}, \ldots, \xi_{k}, \mathbf{0}\right)$ cannot be smaller than $k+1$ as there exist points close to $\mathbf{0 f}$ where the $k+1$ vector fields $\xi, \xi_{1}, \ldots, \xi_{k}$ are independent.

It follows from Lemmas 3 and 4 that the vector fields $\xi_{\epsilon}, \xi_{1}, \ldots, \xi_{k}$ belong to $L_{z}$, for small nonzero $z \in \gamma\left(\xi_{\epsilon}\right)$, for an open set of regular values of $\epsilon$. 
This implies that there exists an open subset $U \in B\left(\xi, \xi_{1}, \ldots, \xi_{k}\right)$ such that $\pi$ has rank $k+1$ at each point of $U$, the set $\pi U$ is a $(k+1)$-dimensional analytic submanifold in $\mathbb{C}^{n}$ with $\mathbf{0} \in \overline{\pi U}$, and the vector fields $\xi, \xi_{1}, \ldots, \xi_{k}$ are tangent to $\pi U$. Hence the brackets of all orders of these vector fields are tangent to $\pi U$ as well.

If $d=d\left(\xi, \xi_{1}, \ldots, \xi_{k}\right)>k+1$ then there exist $k+2$ vector fields from $\mathcal{L}\left(\xi, \xi_{1}, \ldots, \xi_{k}\right)$ that are independent at $\mathbf{0}$. As $\pi U$ is $(k+1)$-dimensional, these vector fields cannot be all tangent to $\pi U$. This contradiction proves that $d=k+1$.

Proposition 1. Let $\Xi=\left\{\xi_{i}\right\}$ be a set of germs at $\mathbf{0} \in \mathbb{C}^{n}$ of analytic vector fields such that $\xi_{1}(\mathbf{0}) \neq 0$, and let $f$ be a germ of an analytic function in $\mathbb{C}^{n}$, with $f(\mathbf{0})=0$, which does not vanish identically on $\gamma\left(\xi_{1}\right)$. Let $d=d(\Xi$, $\mathbf{0})$. There exist $d$ vector fields $\chi, \chi_{1}, \ldots, \chi_{d-1}$ where

a) $\chi=\xi_{1}, \chi_{1}$ is one of $\xi_{i}$, and $\chi_{k}$, for $1<k<d$, is either one of $\xi_{i}$ or belongs to the linear subspace generated by $\left[\chi_{\mu}, f \chi_{\nu}\right]$, for $\mu, \nu<k$, and the following properties are valid for $k=1, \ldots, d-1$ :

$\left.b_{k}\right)$ The system $\left\{\chi, \chi_{1}, \ldots, \chi_{k}\right\}$ is non-degenerate, and a regular value $\epsilon=\left(\epsilon_{1}, \ldots, \epsilon_{k}\right)$ with $\epsilon_{k}=0$ can be found.

$\left.c_{k}\right)\left.\chi \wedge \chi_{1} \wedge \ldots \wedge \chi_{k}\right|_{\gamma\left(\chi_{\epsilon}\right)} \not \equiv 0$, for all values of $\epsilon=\left(\epsilon_{1}, \ldots, \epsilon_{k}\right)$ in an open semi-algebraic set in $\mathbb{C}^{k}$, and a value of $\epsilon$ satisfying this property with $\epsilon_{k}=0$ can be found. Here $\chi_{\epsilon}=\chi+\epsilon_{1} \chi_{1}+\ldots+\epsilon_{k} \chi_{k}$.

Proof. Starting with $\chi=\xi_{1}$, we want to define the vector fields $\chi_{k}$ inductively in $k$. Suppose that, for $k<d$, the vector fields $\chi_{1}, \ldots, \chi_{k-1}$ satisfying a), $\mathrm{b}_{k-1}$ ), and $\mathrm{c}_{k-1}$ ) are already found. We want to find a vector field $\chi_{k}$ such that the system $\left\{\chi, \chi_{1}, \ldots, \chi_{k}\right\}$ satisfies $\left.\left.a\right), b_{k}\right)$, and $c_{k}$ ).

Let $B_{k-1}=B\left(\chi, \chi_{1}, \ldots, \chi_{k-1}\right)$. There are two possible cases. Suppose first that

$$
\chi(z), \chi_{1}(z), \ldots, \chi_{k-1}(z) \text { belong to } L_{z}=\pi T_{z, \epsilon} B_{k-1}
$$

where $z \in \gamma\left(\chi_{\epsilon}\right)$ is a small nonzero point, and $\epsilon$ is any regular value. This is always the case when $k=1$. Due to Lemma 5 , there exists a germ of a $k$ dimensional integral manifold $Y$ for $\chi, \chi_{1}, \ldots, \chi_{k-1}$ at $\mathbf{0}$. As $k<d=d(\Xi$, $\mathbf{0})$, there exists a vector field $\xi_{i} \in \Xi$ such that $\left.\xi_{i}\right|_{Y}$ is not identically tangent to $Y$. In this case, we define $\chi_{k}=\xi_{i}$.

Due to inductive hypothesis, the vector fields $\chi, \chi_{1}, \ldots, \chi_{k-1}$ are independent at small nonzero $z \in \gamma\left(\chi_{\epsilon}\right) \subset Y$, for $\epsilon=\left(\epsilon_{1}, \ldots, \epsilon_{k-1}\right)$ outside an 
open semi-algebraic set in $\mathbb{C}^{k-1}$. As all these vector fields are tangent to $Y$, and $\left.\chi_{k}\right|_{Y}$ is not identically tangent to $Y$, the vector fields $\chi, \chi_{1}, \ldots, \chi_{k}$ are independent at a generic point of $Y$. The same arguments as in the proof of Lemma 3 show that the set of values $(\epsilon, \delta) \in \mathbb{C}^{k}$ for which $\chi, \chi_{1}, \ldots, \chi_{k}$ are independent at small nonzero points of $\gamma\left(\chi_{\epsilon}+\delta \chi_{k}\right)$ is an open semi-algebraic set. In our case, the intersection of this set with $\delta=0$ is non-empty. Hence the property $\mathrm{c}_{k}$ ) is valid for our $k+1$ vector fields.

Let us check the property $\left.\mathrm{b}_{k}\right)$. Let $\epsilon=\left(\epsilon_{1}, \ldots, \epsilon_{k-1}\right)$ be a regular value for the non-degenerate system $\left\{\chi, \chi_{1}, \ldots, \chi_{k-1}\right\}$ such that $\chi_{k}$ is not tangent to $Y$ at small nonzero $z \in \gamma\left(\chi_{\epsilon}\right)$. We claim that $(\epsilon, 0)$ is a regular value for the system $\left\{\chi, \chi_{1}, \ldots, \chi_{k}\right\}$.

We can choose a coordinate system where $\chi_{\epsilon}=\partial / \partial x_{1}$ and $Y=\left\{x_{k+1}=\right.$ $\left.\ldots=x_{n}=0\right\}$. Let $z=(t, 0, \ldots, 0) \in \gamma\left(\chi_{\epsilon}\right)$. According to Lemma 2, there exists a tangent vector $(\zeta(t), 0, \ldots, 0,1)$ to $B_{k}=B\left(\chi, \chi_{1}, \ldots, \chi_{k}\right)$ at a point $(z, \epsilon, 0)$, where $\zeta(0)=0$ and $d \zeta(t) / d t=\chi_{k}$. As $\chi_{k}$ is not tangent to $Y$ at small nonzero $z=(t, 0, \ldots, 0)$, the same is true for $\zeta(t)$. In particular, $\zeta(t)$ does not belong to $L_{z}$ for these values of $z$. As $\epsilon$ is a regular value for $\left\{\chi, \chi_{1}, \ldots, \chi_{k-1}\right\}$, the space $L_{z}$ is $k$-dimensional, for small nonzero $z=(t, 0, \ldots, 0)$. As $\pi T_{z, \epsilon, 0} B_{k}$ contains both $L_{z}$ and $\zeta(t)$, it is $(k+1)$ dimensional, i.e., $(\epsilon, 0)$ is a regular value for $\left\{\chi, \chi_{1}, \ldots, \chi_{k}\right\}$.

Suppose now that (8) is not valid for a regular value of $\epsilon \in \mathbb{C}^{k-1}$, i.e., a generic linear combination $\tau$ of the vector fields $\chi, \chi_{1}, \ldots, \chi_{k-1}$ does not belong to $L_{z}$, for small nonzero $z \in \gamma\left(\chi_{\epsilon}\right)$. As $f$ does not vanish identically on $\gamma(\chi)$, we can choose $\chi_{\epsilon}$ so that $f$ does not vanish identically on $\gamma\left(\chi_{\epsilon}\right)$. Due to Lemma 4 , we can suppose that $\left[\chi_{\epsilon}, \tau\right]$ does not belong to the subspace generated by $\chi, \chi_{1}, \ldots, \chi_{k-1}$ at small nonzero $z \in \gamma\left(\chi_{\epsilon}\right)$. In this case, we define $\chi_{k}=\left[\chi_{\epsilon}, f \tau\right]=f\left[\chi_{\epsilon}, \tau\right]+d f\left(\chi_{\epsilon}\right) \tau$. As $f$ does not vanish identically on $\gamma\left(\chi_{\epsilon}\right)$, the vector fields $\chi, \chi_{1}, \ldots, \chi_{k}$ are independent at small nonzero points of this trajectory. The same arguments as in the proof of Lemma 3 show that the set of values $(\epsilon, \delta) \in \mathbb{C}^{k}$ for which $\chi, \chi_{1}, \ldots, \chi_{k}$ are independent at small nonzero points of $\gamma\left(\chi_{\epsilon}+\delta \chi_{k}\right)$ is an open semialgebraic set. Hence the property $\mathrm{c}_{k}$ ) is valid for these $k+1$ vector fields.

As $f(\mathbf{0})=0$, the property $\left.\mathrm{b}_{k}\right)$ follows from Lemma 2 , as a vector $\left(\zeta_{k}(z), 0, \ldots, 0,1\right)$ is tangent to $B_{k}$ at $(z, \epsilon, 0)$, for small nonzero $z \in \gamma\left(\chi_{\epsilon}\right)$, with $\zeta_{k}=f \tau$. As $\tau(z)$ does not belong to $L_{z}$, for small nonzero $z \in \gamma\left(\chi_{\epsilon}\right)$, the value $(\epsilon, 0)$ is regular for the system $\left\{\chi, \chi_{1}, \ldots, \chi_{k}\right\}$.

Proposition 2. In the conditions of Proposition 1, let vector fields $\xi_{i}$ be 
polynomial, of degree not exceeding $p$, and let $f$ be a linear function. Then each vector field $\chi_{k}$ is polynomial of degree not exceeding $2^{k-1} p$.

Proof. The statement follows from the property a) in Proposition 1.

Definition 4. A subring $\mathbf{S}$ of the ring $\mathbb{R}$ of real numbers is called computable if

(1) each $a \in \mathbf{S}$ can be defined by a finite expression;

(2) for each two numbers $a$ and $b$ in $\mathbf{S}$, there exists a procedure that allows one to derive expressions for $a-b$ and $a b$ from expressions for $a$ and $b$;

(3) there exists a procedure that allows one to decide, for a number $a \in \mathbf{S}$ defined by a given expression, whether $a>0, a<0$, or $a=0$ (see [17]).

Theorem 3. Let $\Xi=\left\{\xi_{i}\right\}$ be a system of vector fields in $\mathbb{C}^{n}$ or $\mathbb{R}^{n}$ with polynomial coefficients of degree not exceeding $p \geq 1$. For $z \in \mathbb{C}^{n}$, let $d=d(\Xi, z)$. The degree of nonholonomy of $\Xi$ at any point $z$ does not exceed

$$
\begin{cases}(d-2)\left(2^{3 d-7} 5 p^{3}\right)^{2^{n-2}}+d-1, & \text { for } d \geq 3 \\ \left(6 p^{3}\right)^{2^{n-2}}+1, & \text { for } d=2 .\end{cases}
$$

Proof. We can take $z=\mathbf{0}$. Let $\chi_{0}=\chi, \chi_{1}, \ldots, \chi_{d-1}$ be the polynomial vector fields defined in Proposition 2. Due to condition $\mathrm{c}_{d-1}$ ) of Proposition $1, Q=\chi_{0} \wedge \ldots \wedge \chi_{d-1}$ does not vanish identically on $\gamma\left(\chi_{\epsilon}\right)$, for some $\epsilon=\left(\epsilon_{1}, \ldots, \epsilon_{d-2}, 0\right)$. Here $\chi_{\epsilon}=\chi_{0}+\epsilon_{1} \chi_{1}+\ldots+\epsilon_{d-2} \chi_{d-2}$. We have

$$
Q=\sum_{i_{1}, \ldots, i_{d}} Q_{i_{1} \ldots i_{d}} \frac{\partial}{\partial z_{i_{1}}} \wedge \ldots \wedge \frac{\partial}{\partial z_{i_{d}}}
$$

where $Q_{i_{1} \ldots i_{d}}$ are polynomials of degree not exceeding $2^{d-1} p$. Due to property a) of Proposition $1, \chi_{\epsilon}$ is a polynomial vector field of degree not exceeding $p$, for $d=2$, and $2^{d-3} p$, for $d \geq 3$. Due to Theorem 1 , this implies that the multiplicity $\kappa$ of restriction of $Q$ to $\gamma\left(\chi_{\epsilon}\right)$ does not exceed the maximum of $\left(6 p^{3}\right)^{2^{n-2}}$ (for $\left.d=2\right)$ and

$$
\left(2^{d-3} p\right)^{2^{n-2}}\left(2^{d-1} p\right)^{2^{n-2}}\left(2^{d-3} 5 p\right)^{2^{n-2}}=\left(2^{3 d-7} 5 p^{3}\right)^{2^{n-2}}
$$

Each derivation of $Q$ along $\chi_{\epsilon}$ decreases this multiplicity by 1 . Hence the result of $\kappa$ consecutive derivations of $Q$ along $\chi_{\epsilon}$ does not vanish at $\mathbf{0}$. 
From the property a) of Proposition 1, each $\chi_{k}$ is a linear combination with polynomial coefficients of brackets of the vector fields $\xi_{i}$ of order not exceeding $d-1$, and $\chi_{\epsilon}$ is a combination of brackets of order not exceeding $\max (1, d-2)$. Taking into account the derivation formula

$$
L_{\chi_{\epsilon}}\left(\chi_{0} \wedge \ldots \wedge \chi_{d-1}\right)=\sum_{i=0}^{d-1} \chi_{0} \wedge \ldots \wedge\left[\chi_{\epsilon}, \chi_{i}\right] \wedge \ldots \wedge \chi_{d-1}
$$

we obtain the estimate (9).

Corollary. If a system $\Xi$ of vector fields in $\mathbb{C}^{n}$ or $\mathbb{R}^{n}$ is finite, $\Xi=\left\{\xi_{i}, i=\right.$ $1, \ldots, I\}$, and if the coefficients of the vector fields in $\Xi$ are polynomials of degree not exceeding $p$ with coefficients from a computable ring $\mathbf{S}$ (Definition 4), there exists an algorithm which allows one to decide whether the system $\Xi$ is totally nonholonomic (controllable), i.e., $d(\Xi, z)=n$ at each point $z$. The complexity of this algorithm can be effectively estimated in terms of the integer numbers $I, n$, and $p$ and the complexity of computations in the ring $\mathbf{S}$.

Proof. According to Theorem 3, the system $\Xi$ is totally nonholonomic if and only if the following system of polynomial equations is incompatible:

$$
\chi_{i_{1}} \wedge \ldots \wedge \chi_{i_{n}}=0, \quad \text { for } 1 \leq i_{1}<\ldots<i_{n} \leq M
$$

Here $\chi_{j}=\left[\xi_{j_{1}} \ldots\left[\xi_{j_{k-1}}, \xi_{j_{k}}\right] \ldots\right]$, with $1 \leq j_{\nu} \leq I$, is a bracket of vector fields from $\Xi$ of the order $k$ not exceeding maximal possible degree of nonholonomy in (9) with $d=n$, and $M$ is the total number of such brackets. The algorithm [17] allows one to check the consistency of a system of algebraic equations with computable coefficients. The estimate of complexity follows from the estimate (9) and the estimate of complexity of the algorithm [17].

Theorem 4. Let $f_{1}(y), \ldots, f_{m}(y)$ be a Noetherian chain (Definition 1) in $\mathbb{C}^{n}$ or $\mathbb{R}^{n}$ of order $m$ and degree $\alpha \geq 1$. Let $\Xi=\left\{\xi_{i}\right\}$ be a set of vector fields with Noetherian coefficients:

$$
\xi_{i}=\sum_{j} P_{i j}\left(y, f_{1}(y), \ldots, f_{m}(y)\right) \frac{\partial}{\partial y_{j}}
$$


with $P_{i j}$ polynomial in $y$ and $f$ of degrees not exceeding $p \geq 1$. Let $d=$ $d(\Xi, \mathbf{0})$. The degree of nonholonomy of $\Xi$ at $\mathbf{0}$ does not exceed

$$
\begin{cases}(d-2)\left(2^{3 d-7} 5(p+\alpha)^{3}\right)^{2^{m+n-2}}+d-1, & \text { for } d \geq 3, \\ \left(6(p+\alpha)^{3}\right)^{2^{m+n-2}}+1, & \text { for } d=2 .\end{cases}
$$

Proof. Consider a submanifold $Y=\left\{f_{i}=f_{i}(y)\right\}$ in $\mathbb{C}_{y, f}^{n+m}$ with a natural projection $\pi: Y \rightarrow \mathbb{C}_{y}^{n}$. It is easy to show that the polynomial vector fields

$$
\sum_{j=1}^{n} P_{i j}(y, f) \frac{\partial}{\partial y_{j}}+\sum_{l=1}^{m}\left[\sum_{j=1}^{n} P_{i j}(y, f) g_{l j}(y, f)\right] \frac{\partial}{\partial f_{l}}
$$

are tangent to $Y$ and their restrictions to $Y$ coincide with $\pi^{*} \xi_{i}$. Here $g_{l j}$ are polynomials from the definition (3) of a Noetherian chain. The same is true, of course, for the brackets of any order of these vector fields. The statement follows now from Theorem 3 applied to the polynomial vector fields (11).

Corollary. Suppose that in Theorem 4 the system $\Xi$ is finite, $\Xi=\left\{\xi_{i}, i=\right.$ $1, \ldots, I\}$, and that the values $f_{1}(\mathbf{0}), \ldots, f_{m}(\mathbf{0})$, the coefficients of the polynomials $g_{i j}$ in the definition of the Noetherian chain $f_{1}, \ldots, f_{m}$, and the coefficients of the polynomials $P_{i j}$ belong to a computable ring $\mathbf{S}$ (Definition 4). Then there exists an algorithm which allows one to decide whether the system $\Xi$ is totally nonholonomic (controllable) at $\mathbf{0}$, i.e., $d(\Xi, \mathbf{0})=n$. The complexity of this algorithm can be effectively estimated in terms of the integer numbers $I, n, m, \alpha$, and $p$, and the complexity of computations in the ring $\mathbf{S}$.

Proof. According to Theorem 4, the system $\Xi$ is totally nonholonomic at $\mathbf{0}$ if and only if the following system of polynomial equations with coefficients from $\mathbf{S}$ is incompatible:

$$
\chi_{i_{1}}(\mathbf{0}, f(\mathbf{0})) \wedge \ldots \wedge \chi_{i_{n}}(\mathbf{0}, f(\mathbf{0}))=0, \quad \text { for } 1 \leq i_{1}<\ldots<i_{n} \leq M .
$$

Here $\chi_{j}=\left[\xi_{j_{1}} \ldots\left[\xi_{j_{k-1}}, \xi_{j_{k}}\right] \ldots\right]$, with $1 \leq j_{\nu} \leq I$, is a bracket of the polynomial vector fields $(11)$ in $(n+m)$-dimensional space of the order $k$ not exceeding maximal possible degree of nonholonomy in (10) with $d=n$, and $M$ is the total number of such brackets. 


\section{Acknowledgements}

The author is grateful to J. J. Risler, A. Khovanskii, E. Bierstone, P. Milman, I. Kupka, V. Palamodov, and N. Vorobjov for discussions, and to C. Roche and C. Moura for their valuable remarks on a preliminary version of the manuscript. This work was partially done when the author visited the Department of Mathematics at the University of Toronto.

\section{References}

1. J. P. D'Angelo, Several complex variables and the geometry of real hypersurfaces, CRC Press, 1993.

2. T. Bloom and I. Graham, On 'type' conditions for generic real submanifolds of $\mathbb{C}^{n}$, Invent. Math., 40 1977, 217-243.

3. $\mathbb{C}^{n}$, J. Diff. Geom., 12 1977, 171-182.

4. R. W. Brocket, Nonlinear control theory and differential geometry, Proc. of the Intnl. Congress of Mathematicians, Warsaw, 1983.

5. C. Fefferman and D. H. Phong, Subelliptic eigenvalue problems, Proc. of the Conference on harmonic analysis in honor of Antoni Zygmund. Wadsworth Math. Series 1981, 590-606.

6. A. Gabrielov, J. M. Lion and R. Moussu, Ordre de contact de courbes intégrales du plan, CR Ac. Sci. Paris, 319, 1994, 219-221.

7. A. Gabrielov, Multiplicities of Pfaffian intersections and the Eojasiewicz inequality, preprint 93-64, Cornell MSI, 1993.

8. V. Gershkovich and A. Vershik, Nonholonomic manifolds and nilpotent analysis, J. Geometry and Physics, 5, 1988, 407-452.

9. M. Gromov, J. Lafontaine and P. Pansu, Structures métriques pour les variétés Riemanniennes, Cedic-Fernand Nathan, Paris, 1981.

10. M. Gromov, Carnot-Caratheodory spaces seen from within, preprint IHES, 1994.

11. B. Helffer and J. Nourrigat, Hypoellipticité maximale pour des opérateurs polynomes de champes de vecteurs, Birkhauser, 1985.

12. L. Hörmander, Hypoelliptic second order differential equations, Acta Math., 119, 1968, 147-171.

13. The analysis of linear partial differential operators III, Springer-Verlag, 1985.

14. J. J. Kohn, Boundary behavior of $\bar{\partial}$ on weakly pseudo-convex manifolds of dimension two, J. Diff. Geom., 6, 1972, 523-542.

15. S. Kusuoka and D. Stroock, Application of the Malliavin calculus, Part II, J. Fac. Sci., Univ. Tokyo, 32, 1-76, 1985; Part III, J. Fac. Sci., Univ. Tokyo, 34, 391-442, 1987.

16. A. Nagel, E. M. Stein and S. Wainger, Balls and metrics defined by vector fields I: basic properties, Acta Math., 155, 103-147, 1985.

17. J. Renegar, On the computational complexity and geometry of the first order theory of reals, Parts I-III, J. Symb. Comp., 13, 1992, 255-352.

18. J. J. Risler, A bound for the degree of nonholonomy, preprint LMENS 94-6, 1994.

19. A. Sánchez-Calle, Fundamental solutions and geometry of the sum of squares of vector fields, Inv. Math., 78, 143-160, 1984. 
20. R. S. Strichartz, Sub-Riemannian geometry, J. Diff. Geometry, 24, 1986, 221-263.

21. H. J. Sussmann, Lie brackets, real analyticity and geometric control, in Differential geometric control theory, Brockett et al., Eds., Birkhauser, 1982, 1-116.

22. J.-C. Tougeron, Algèbres analytiques topologiquement nœthériennes, Théorie de Hovanskii, Ann. Inst. Fourier, 41, 823-840, 1991.

23. A. M. Vershik, V. Ya. Gershkovich, Nonholonomic dynamical systems, geometry of distributions and variational problems, in Dynamical systems VII, Arnold and Novikov, Eds., Springer-Verlag, 1994, 1-8.

Mathematical Sciences Institute, Cornell Univerity, 409 College Av., IтнACA, NY 14850

E-mail address: andrei@msiadmin.cit.cornell.edu 